
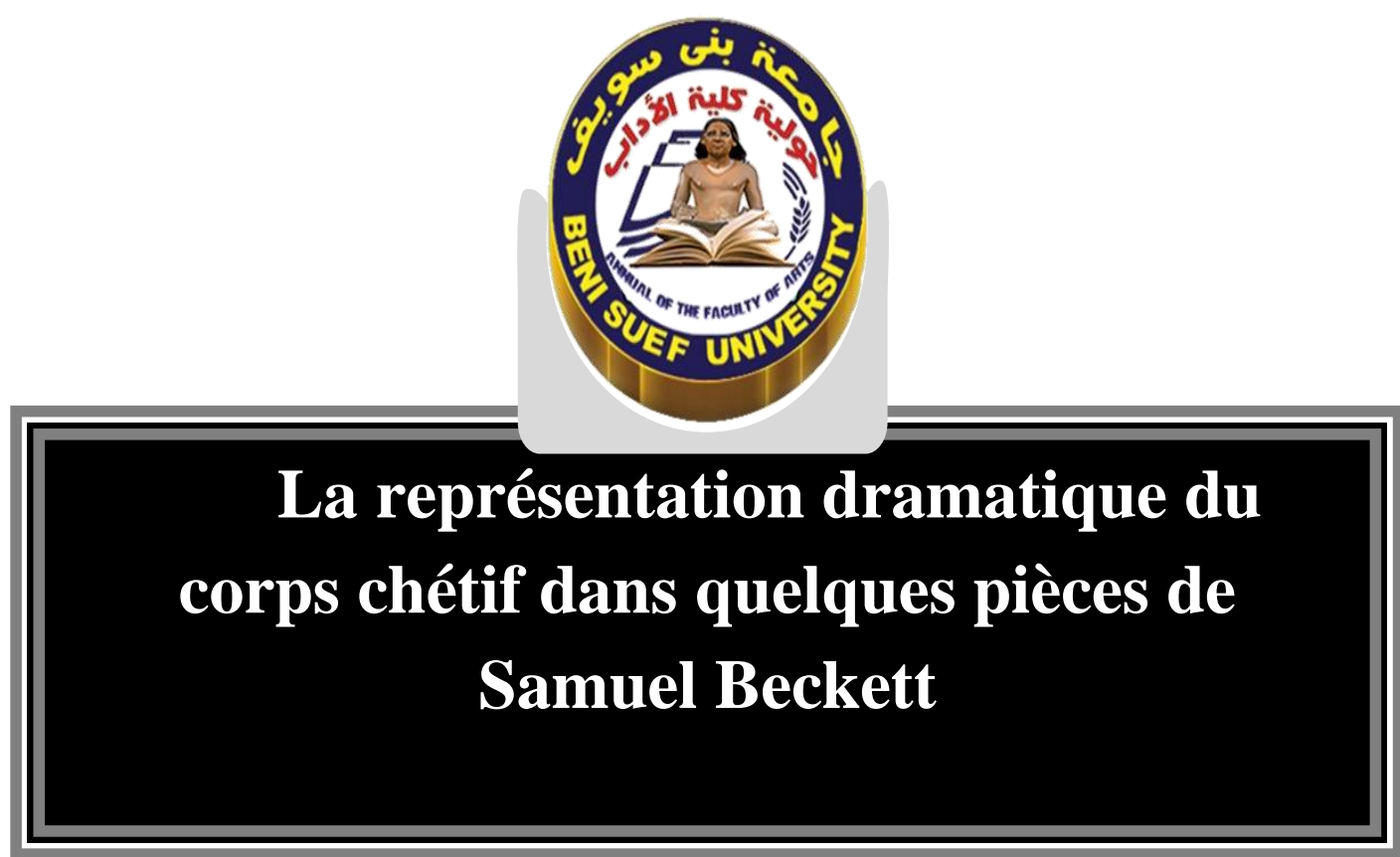

\title{
Préparée par:
}

\section{Dr. Mohamed Abdel Tawab}

Maître de Conférences à la faculté des Lettres

Université du Fayoum

\section{الإستشهاد المر جمى:}

Mohamed Abdel Tawab(2021) .La représentation dramatique حولية - حu corps chétif dans quelques pièces de Samuel Beckett

$$
\text { كلية الآداب. جامعة بني سويف. . مج • 1:ج r. . ص ص .. 9هـ- ؟9هـ }
$$

\section{Abstract:}

Les conséquences terribles qui ont résulté de la Seconde guerre mondiale a mené un groupe d'écrivains, tels Adamov, Ionesco et Beckett, à inventer un nouveau courant littéraire, il s'agit du théâtre de l'Absurde. Leur recul par rapport à la langue française leur a donné une perception aiguë de 
l'incommunicabilité entre les êtres. Ce sentiment qui a fait le tragique dans la modernité occidentale.

Samuel Beckett est l'un des dramaturges les plus célèbres au vingtième siècle qui ont bien représenté cette incommunicabilité et cette souffrance entre les êtres à travers le corps sur l'espace scénique. Selon la pensée de Beckett, il n'y a que le corps, seul moyen, qui lui permettait de transmettre son message et d'atteindre finalement le public. Dans le monde beckettien, le corps représente d'ailleurs toute forme de torture, d'exploitation et d'oppression de la part des hommes belliqueux.

Le corps devient donc l'incarnation de toutes les souffrances physiques et morales qui dénue l'homme de son humanité et de son identité. Ce serait le reflet de toute l'angoisse existentielle et misérable humaine engendrée la guerre. C'est pour cela que les pièces beckettiennes s'inscrivent dans une forme tragique et absurde.

descriptors

Corps chétif- absurdité - Samuel Beckett

"'Ce soir, voyant cet univers absurde, insignifiant et néant. Un monde où l'existence est impossible, où les maladies et l'effondrement sont partout. Mon corps, mon vieux corps s'effondre et disparaît, ainsi qu'un morceau de glace se fond sous l'impact d'un coup de massue. Il $n$ 'en reste que son image déchéante, qui serait finalement l'image de son être chétif' '(Beckett, le Calmant, 1956, p. 45) 


\section{Introduction de recherche:}

Les conséquences terribles qui ont résulté de la Seconde guerre mondiale a mené un groupe d'écrivains, tels Adamov, Ionesco et Beckett, à inventer un nouveau courant littéraire, il s'agit du théâtre de l'Absurde. Leur recul par rapport à la langue française leur a donné une perception aiguë de l'incommunicabilité entre les êtres. Ce sentiment qui a fait le tragique dans la modernité occidentale.

Samuel Beckett est l'un des dramaturges les plus célèbres au vingtième siècle qui ont bien représenté cette incommunicabilité et cette souffrance entre les êtres à travers le corps sur l'espace scénique. Selon la pensée de Beckett, il n'y a que le corps, seul moyen, qui lui permettait de transmettre son message et d'atteindre finalement le public. Dans le monde beckettien, le corps représente d'ailleurs toute forme de torture, d'exploitation et d'oppression de la part des hommes belliqueux.

Le corps devient donc l'incarnation de toutes les souffrances physiques et morales qui dénue l'homme de son humanité et de son identité. Ce serait le reflet de toute l'angoisse existentielle et misérable humaine engendrée la guerre. C'est pour cela que les pièces beckettiennes s'inscrivent dans une forme tragique et absurde.

\section{- Motif du Choix}

Le corps est une donnée essentielle de la condition humaine, mais il est rarement pris en considération par la littérature et notamment le genre dramatique. En effet, il est très remarquable qu'il y a très peu de personnages qui sont malades ou souffrants en littérature. Même si Molière met en scène Aragon, il n'est enfin de compte qu'un « malade imaginaire ». Donc, la singularité de Beckett réside dans le fait que les personnages représentent leur infirmité sur scène et parlent sans gêne de 
leurs maux, sans le dire explicitement. Cette singularité se laisse percevoir aussi dans la détérioration de tous les éléments fondateurs de la représentation comme la décomposition du corps, par exemple. C'est en cela que réside sa nouveauté. Toutes ces raisons nous ont amené à consacrer une recherche sur l'importance du corps dans le théâtre français.

\section{- Problématique de la recherche}

Cet article se propose d'analyser les principales formes de représentation dramatique du corps sur l'espace scénique d'après l'œuvre dramatique de Samuel Beckett. Cette dernière y donne une place dominante dans son théâtre sur tout à partir des années cinquante. Or, le corps qu'il représente n'est pas celui-ci que tout le monde perçoit. C'est le corps dépourvu de ses fonctions, c'est le corps infirme, immobile, handicap, cul- de jatte et, comme le souligne le titre de l'article, chétif. Cette mutilation du corps est très représentative dans son univers théâtral. Elle lui permet de faire part de sa vision pessimiste de la vie et de montrer la réalité humaine, à travers la souffrance des personnages. Ces derniers constituent un système signifiant de la vie et de ses maux.

Selon la pensée beckettienne, la représentation dramatique d'un corps est porteuse d'un regard désenchanté sur la situation du monde d'après la guerre, c'est pour cela que c'est une donnée essentielle dans la littérature française et le théâtre français en particulier.

Le corps représente pour Beckett l'acteur principal et l'enjeu de toute la problématique théâtrale beckettienne. Il est donc un personnage principal au même titre que les autres personnages de la pièce. Il devient l'intrigue même de la pièce. Il constitue pour les spectateurs à la fois le point de départ et d'aboutissement pour eux. Il sera ensuite l'objet d'une quête permanente 
Soulignons aussi que cette représentation prend plusieurs formes : une fois, elle prend la forme d'un corps mutilé, d'un corps paralysé, d'un corps qui souffre moralement et physiquement, d'un corps cul- de jatte qui est au point de mettre fin à sa vie, d'un corps mis en scène, un corps borgne et malvoyant. Toutes ces formes de difformité du corps feront l'objet de notre étude et notre traitement dans la recherche.

En gros, Beckett a recours à cette technique dramatique du corps pour renforcer le côté tragique et le rendre différent en forme et en norme. Or, c'est en cela que réside son originalité.

Nous allons tenter dans cet article de faire preuve de sa vision pessimiste du monde et de savoir pourquoi il a adopté l'immobilité et la difformité comme éléments principaux dans son théâtre. De surcroit, nous allons essayer de répondre, par le biais de la méthode psychanalytique, à la question suivante: pourquoi a-t-il mis les personnages dans des situations burlesques et tragiques en se penchant sur ses pièces autour duquel se focalise le côté tragique et comique?

\section{- Le corpus}

1-Samuel Beckett, En attendant Godot, Editions de Minuit, 1953

2-Samuel Beckett, Fin de Partie, Editions de Minuit, 1957

3-Samuel Beckett, Oh! Les beaux jours, Editions de Minuit, 1963

\section{- La méthode de la recherche}

Dans cette recherche nous allons adopter dans la méthode psychanalytique, seule méthode permettant d'expliciter et, plus précisément du résultat de l'effet des complexes psychiques que créé l'impotence du corps sur la vie quotidienne des personnages (les regards, les déplacements, la vie sociale, les gestes et les vêtements, les 
conditions de vie) De surcroît, nous allons tenter d'entrer dans la vie psychique dans l'inconscient de chaque personnage pour dégager l'effet de cette souffrance physique et morale d'après la représentation théâtrale. Cette analyse psychologique nous permet de découvrir les blessures de leur condition de misérables et le reflet de cette difficulté à survivre face à' l'absurdité'" de la vie. Pour ce faire, nous opterons pour un corpus de trois pièces où le handicap et l'impuissance physique et morale constituent l'objet principal de notre analyse dans le cadre de cette étude.

\section{1- le corps est un être qui souffre}

Une des techniques dramatiques de Beckett, c'est qu'il nous représente un corps qui souffre et qui se décompose jusqu' à la mort. Sa mise en scène d'un corps mutilé et impotent constitue une de ses esthétiques de son théâtrales comme le montre cet extrait suivant: "Sa fascination pour la décomposition des corps et de la mort le rend proche des favoris du mal et de l'horreur" (Grossman, Novembre 1998, p. 19).Ses œuvres dramatiques en sont un exemple:En attendant Godot, les chutes macabres de Pozzo et de Lucky; la démarche difficile et tremblante de Clov dans Fin de partie et enfin l'avancement malaisé de Willie sur sa face ventrale dans Oh! les beaux jours. Si nous faisons un regard sur l'œuvre dramatique de Beckett, nous trouvons que ses personnages font toujours l'objet d'une déchéance physique jusqu' à devenir handicapés ou assis sur des fauteuils à roulants, à savoir, le corps chez lui est toujours caractérisé par l'immobilité et la paralysie. De plus, la difficulté de Hamm à porter ses lunettes d'aveuglement, d'une part, et de Winnie à chercher sa loupe pour lire et de l'ajuster de temps en temps pour pouvoir en avoir facilement, d'autre part, met en évidence la souffrance de l'être humain incarné dans le corps, nous pouvons l'éprouver d'après cet 
extrait tiré d'Oh! Les beaux jours :"Winnie cherche la loupe afin de la tourner sur la carte. Elle dépose la loupe, plus loin, elle les chausse" (Beckett, Oh!les beaux jours, 1963, p. 21).

Mais, à la différence des dramaturges français, Beckett a rendu ses personnages voir cette impotence comme signe de joie et de plaisir. Selon lui, c'est dans cette immobilité que les personnages trouvent aide et soutien. Cette paralysie leur permet d'être toujours à l'écart du monde matériel dans lequel ils trouvent panique et angoisse. Voici un extrait, tiré d'Oh! les beaux jours, qui le prouve :

"Quelle malédiction la mobilité! (Beckett, Oh!les beaux jours, 1963, p. 33)

Il en va de la sorte pour Hamm, personnage à la fois omnipotent, régnant en maître des mots et des actions, condamné à l'inertie et à la cécité mène une vie normale.

Autre scène qui montre que le corps souffrant est un composant fondamental dans l'univers théâtral de Beckett et que c'est quelque chose de normal :",

"Willie. Malgré son immobilité, son infirmité, il avance à quatre pattes vers le centre, s'arrête, tourne la tête de force"' (Beckett, Oh!les beaux jours, 1963, p. 34)

Bien que le corps soit malade, vieilli, impotent, torturé et paralytique, il parait naturel comme si rien ne s'y passait, comme s'il s'agissait de quelque chose d'ordinaire, de prédestiné. Nous pouvons le constater à travers cet extrait :

"Hamm : comment vont tes jambes?

Clov : mal

Hamm : mais tu peux bouger? 


\section{Dr. Mohamed Abdel Tawab}

Clov : oui " (Beckett, Fin de Partie, 1957, p. 22)

La dualité entre le corps et ses organes, entre le corps et ses possibilités a pour objectif de décomposer les éléments pathétiques et les égalisent en les faisant passer pour quelque chose d'ordinaire.

D'autre part, les mouvements répétés de chaque personnage n'ont d'autres choses qu'une autre forme de souffrance. Le procédé de répétition fait par les personnages produit des effets décisifs sur le statut et la signification du texte. Prenons par exemple, En attendant Godot qui débute par une répétition gestuelle qui indique d'emblée la teneur physique du jeu des acteurs :"Estragon, assis sur une pierre, essaie d'enlever sa chaussure. Il s'y acharne des deux mains, en ahanant. Il s'arrête, à bout de forces, se repose en haletant, recommence. Même jeu. Cette répétition est suivie d'une autre, celle de Vladimir qui par deux fois ôte son chapeau, regarde dedans, y promène sa main, le secoue, le remet" (Beckett, En attendant Godot, 1953, p. 19).Cette réitération n'a qu'à donner sens à l'action dramatique de la pièce. Ce procédé expose les fonctions de ces objets a son seul profit : la chaussure et le chapeau acquièrent une équivalence par le même geste qui s'y applique, comme le dit Michèle Fourré "il n'y a rien à découvrir, ni l'un ni dans l'autre, et c'est la répétition elle-même qui se donne à voir" (Foucré, 1970, p. 67). Pour sa part, Beckett rappelle à ses metteurs en scène l'importance de ces répétitions dont ils recommandent d'observer les effets. En même temps, ce système répétitif vise aussi à mettre en évidence la souffrance physique et morale que subissent les personnages au sens où l'entend Artaud d'une "cruauté physique" (Artaud, 1966, p. 54) de Beckett. Donc, nous pouvons dégager que la répétition des actes sur l'espace scénique est l'un des éléments constitutifs de la représentation du corps. 
Autre forme de la souffrance physique du corps, c'est la dépendance de de l'Autre. La forme du couple beckettien est considérée comme l'une des techniques dramatiques qui ont aliéné Beckett dans son univers théâtral.

Chacun ne peut jamais se passer de l'autre, ils sont comme l'ombre et le corps et que toute idée de séparation entre les personnages est presque impossible :"la vie de Hamm alterne, grâce à Clov, le lever et le coucher" (Ryngaert, 1993, p. 29). Le fait de se quitter est le point de départ pour une autre vie; il s'agit de la mort. Comme le montre cette citation :

Clov dit à Hamm :"j'ai mal aux jambes,

Hamm réplique : avec soulagement : tu ne peux pas me quitter" (Beckett, Fin de Partie, 1957, p. 69)

Comme le montre cet extrait que le dramaturge met en scène un personnage qui marche avec difficulté, c'est pour cela qu'il demande un coup de main d'un autre pour pouvoir se déplacer avec facilité. C'est le cas de Clov, un personnage handicap qui se véhicule sur un fauteuil à roulettes, Hamm son Co-assistant lui dit "tu te rappelles, au début, quand tu me faisais ma promenade, comme tu t'y prenais mal ? A chaque pas tu manquais de me verser" (Beckett, Fin de Partie, 1957, p. 89).

Chaque couple présente une position et son contraire, puisque l'un ne peut se tenir debout, tandis que l'autre ne peut s'asseoir. Quoi qu'il en soit, Hamm et Clov ne peuvent se séparer.

Selon la pensée beckettienne, ces êtres sadiques, qui vivent avec leurs infirmités et leurs immobilités, passent la majorité de leur temps à se faire souffrir. 
D'où l'importance de l'aliénation de la dépendance selon la vision de Beckett.

D'autre part, autre la dégradation physique des personnages, Beckett leur a présentés à une faiblesse morale incarnée dans la faiblesse sexuelle et l'incapacité de faire l'amour ensemble à cause de l'angoisse existentielle de la vie :

"Nagg : embrasse

Nell : on nepeut pas

Nagg : essayons" (Beckett, Fin de Partie, 1957, p. 23)

Comme le montre cet extrait, Nagg et Nell sont deux personnages qui trouvent des difficultés à se coucher et à passer des moments heureux ensemble. Tous les deux vivent enterrés et isolés dans deux poubelles en s'éloignant l'un de l'autre. Par conséquent, ils ne se communiquent pas.

En plus, Winnie qui, dans Oh !les beaux jours, supplie son mari Willie de l'embrasser en ces termes:

Allons, mon cœur, du nerf, vas-y,

Je t'applaudirai" (Beckett, Oh!les beaux jours, 1963, p. 44) mais il lui dit qu'il ne peut pas.

De surcroît, tout le monde souffre de l'incommunicabilité et de l'isolement d'avec l'autre, c'est le rythme dominant chez Pozzo qui, En attendant Godot, ne cesse d'appeler au secours à cause de la solitude dans laquelle il vit.

Il nous semble que cette déformation du corps ainsi que cette souffrance physique et morale sont dues à des souvenirs autobiographiques chez Beckett : les années d'enfance qu'il a passées en Irlande étaient très difficiles : abcès, grippes et douleurs articulaires. 
Par ailleurs, le thème de dépendance mutuelle du couple chez Beckett a influencé un bon nombre de grands dramaturges tels Bernard Liègme, et Gilbert Léautier. Évoquons par exemple, dans Le Borgne est roi de (Fuentès, 1972, p. 3), Les deux aveugles sont à la fois grotesques et pitoyables. Les deux hommes sont aliénés. Ils ne peuvent pas se passer l'un de l'autre en prenant pour exemple les héros beckettiens.

La dépendance du couple beckettien est aussi une source d'inspiration dans Tandem (Liègme, 1976). Dans cette pièce, il est question d'un vieil infirme qui s'attache désespérément à sa femme pour marcher. Il en est de même pour l'héroïne de la Matriarche (Léautier, 1978). Il s'agit d'une vieille grand-mère aveugle et sourde.

Nous percevons aussi l'influence du couple Hamm-Clov de Beckett chez le couple Dodge (Dodge, 1968), un vieillard qui ne peut plus se lever de son lit sans l'aide de son fils, un type complètement demi-fou.

D'autre part, Fragment du théâtre (Beckett, fragment du theatre I et II, 1978) dernière pièce de Beckett constitue la dernière pièce où le corps est représenté sous cette forme de paralysie. Beckett nous y présente la rencontre de deux personnages, l'un aveugle et l'autre paralytique. La technique dramatique de Beckett réside justement dans cette infirmité de chacun d'eux. Chaque homme présage l'espoir dans cette immobilité qui est censée rendre sa joie et le récompenser de son infirmité. 


\section{2- Le corps est mis en scène}

C'est à partir de 1960 que la représentation du corps commence à prendre une nouvelle forme. Il n'est plus l'infirmité du corps qui est centrale dans le théâtre de Beckett, mais encore plutôt l'image du corps qui le préoccupe et l'intéresse plus que tout. Selon lui, le théâtre d'art visuel permet aux spectateurs d'atteindre son message facilement. De plus, Bien que le corps souffre et se dégrade physiquement et moralement. Il est quand même visuel. Il nous montre un corps morcelé qui va plus loin jusqu'à l'anéantissement et la décomposition entières. Donc, nous pouvons dégager que la représentation de l'image du corps devient plus importance que son souffrance physique et morale.

De ce point de vue, Oh!les beaux jours, marque un changement dans le parcours dramatique de Beckett. Winnie, au fil du temps, est engloutie dans son mamelon, puis un tronc et finalement devient une tête. Beckett note à l'acte II:

«La tête, qu'elle ne peut plus tourner, ni lever,

ni baisser, reste rigoureusement immobile et de face » (Beckett, Oh!les beaux jours, 1963, p. 70)

Winnie se plaint douloureusement de sa décomposition du corps qui lui échappe de toute vue d'une part, et la prive de se communiquer avec son ami, d'autre part. Cet anéantissement la rend toujours à la merci de son compagnon.

Toujours dans cette image de décomposition du corps, les deux personnages, Winnie et Willie, enfermés dans deux jarres, sont réduits à deux visages dans l'acte II d'Oh !les beaux jours.

Cet anéantissement du corps sur l'espace scénique ne vise autre chose qu'à banaliser cette dramatisation et de perdre son sens aux 
spectateurs comme si était quelque chose de normal. Cette technique de Beckett ne fait qu'à interpréter la difficulté de la condition humaine dans laquelle vivent les personnages.

Dans les années soixante-dix, Beckett est allé plus loin jusqu'à morceler complètement son corps en ne laissant que sa bouche comme signe visuel de sa présence qui n'apparaît que dans le noir et qu'on n'entend de sa bouche que deux mouvements respiratoires qui marquent sa vie ainsi que présence.

Donc nous pouvons comprendre que la vie est rythmée par l'inspiration, puis par quelques secondes expiration, à la quelle succède un cri identique au cri initial.

Comme le dit Beckett dans une entrevue «Avant, il n'y a rien ; après, c'est le retour au néant » (Chabret, 1986, p. N0521).Cela se voit dans sa pièce qui s'intitule Pas moi (Beckett, Pas moi, 1972).

L'exhibition d'un corps souffrant sur l'espace scénique est considérée comme une autre technique de l'extrême théâtralité de Beckett.

D'autre part, il faut dire que Beckett a été influencé par ce mode de représentation d'un corps morcelé de grand poète et dramaturge espagnol Picasso où on trouve dans son art un tableau, peint durant la guerre d'Espagne représentant des peuples souffrant des corps mutilés en lambeaux et en pièces à cause d'une explosion quelconque.

Aussi dans Le Désir attrapé par la queue (Picasso, 1964, p. 45) du même dramatique, nous y voyons l'angoisse existentielle et terrifiante de la guerre mondiale par le biais des images en froid qui ne suscitent aucune émotion et qui, au contraire, appelle à la mélancolie et au chagrin 


\section{Dr. Mohamed Abdel Tawab}

et ainsi des personnages qui se plaignent de leurs enflures dans les mains et les pieds.

De plus, Beckett a été influencé aussi par la carrière de Mrozek, un dramaturge polonais du XXIème siècle qui appartient au genre de la fiction absurde qui, dans Striptease (Mrozek, 1961, p. N06), nous met en scène un gardien qui tient de sa propre main deux prisonniers avant de les conduire à la mort, la chose qui reflète l'atrocité et la cruauté de la situation.

Beckett a été influencé aussi par Freud en ce qui concerne sa représentation visuelle du corps sur l'espace scénique. Selon ce psychologue, il y a toujours un grand rapport entre le langage d'un schizophrène et son corps. Ils forment une thématique très importante dans la psychose à savoir le langage de schizophrénie déconstruit tous les éléments discursifs du personnage pour dévoiler l'ancrage du corps. C'est via ce procédé que le personnage exprime son mode de vie et son statut personnel à travers le langage du corps, il s'agit « du langage d'organe » (Freud, 1983, p. 119)

Selon la pensée freudienne, le langage d'organe vise à utiliser des symboles corporels au sein du discours. Selon lui, le langage du corps a la capacité de cristalliser tous les contenus d'un corps malade, d'une part, et de le libérer de ses défoulements, d'autre part, sans le dire. Cela ne vient autrement que par le biais de l'analyse psychologique du patient.

Dans l'univers beckettien, nous remarquons que ce dramaturge a représenté ce phénomène psychique sur le plateau scénique. Il a dissocié le langage d'un malade et le laisse s'exprimer ses maux et son hystérie à travers le langage du corps. Donc, la maladie du corps prend toute l'importance de la scène. 
En gros, représenter le tronc, la bouche ou le reste d'un corps n'est qu'une méthode métonymique pour Beckett pour appeler les spectateurs d'atteindre son message d'après sa vision, d'une part, et de découvrir le côté profond caché de l'inconscient du personnage, d'autre part.

Donc, nous pouvons dégager que Beckett est, à la différence de Picasso et de Mrozek, est unique en son genre. Son théâtre se distingue d'avoir su représenter dramatiquement l'inconscient d'un corps fragmenté.

Par ailleurs, la partie représentée n'est pas tout, puisque le morceau du corps autour duquel se focalise la scène n'est pas saisi. C'est dans ce point que Beckett se montre novateur et touchant dans tout ce qui est inconscient et incompréhensible par le public. La représentation d'un corps décomposé sur la scène n'est qu'un symbole de la fragilité de l'existence et l'absurdité de la vie.

En fin du compte, nous pouvons conclure en disant que le traitement de la scène, dans la dramaturgie beckettien, ne dépend complètement de la mise en scène d'un corps et ce dernier ne peut être appréhendé qu'en le fragmentant.

\section{3- le corps est ravagé par le temps}

Beckett est considéré comme l'un des dramaturges modernes qui ont fait une rupture avec les règles traditionnelles de la dramaturgie classique. C'est à cette notion que joue Beckett sur les multiples dimensions. Il brise les habituels repères qui permettent de représenter le temps, mais il propose une durée singulière que les classiques appelaient l'unité du temps. Les pièces de Beckett remplacent à ce temps théâtral la notion de cours, ébauchée à titre de moteur dans En Attendant Godot et constituée comme substance dans Fin de partie. 


\section{Dr. Mohamed Abdel Tawab}

La stratégie critique du dramaturge moderne consiste à éliminer les références temporelles réalistes en embarrassant systématiquement le spectateur.

Les personnages, vecteurs d'une temporalité humaine, ont été influencés de ce décalage du temps à savoir ils sont enfermés dans un carcan et ne sont pas capables de se situer eux-mêmes au sein de l'action. Ils doutent constamment des actions qu'ils ont pu accomplir mentalement. Le défaut de mémoire qui hante les personnages beckettiens permet de mettre fin aux illusions et les empêcher d'être poursuivies. Dès le début d'En Attendant Godot, Vladimir et Estragon s'interrogent sur ce qui s'est passé la veille : «Qu'est-ce que nous avons fait hier? » (Beckett, En attendant Godot, 1953, p. 67). De surcroit, le doute se répète encore avec le début du deuxième acte, alors que le spectateur est censé être au courant des événements qui ont eu lieu au premier acte.

Une autre forme de l'influence du temps sur le corps, lorsque dans Fin de partie, au début, les personnages évoquent leurs beaux souvenirs et leurs beaux jours. Toutefois avec le temps destructif et répétitif, tout est tombé dans l'oubli. Cela est dû bien sûr à la répétition des événements et la monotonie du temps. Cela se voit lorsque Nagg veut rappeler à Nell l'accident de Tandem où ils sont tombés dans le lac, mais hélas ! Nell n'arrive pas à se rappeler :

"Nagg : tu te rappelles

Nell : Non

Nagg : l'accident de Tandem où nous laissâmes nos guibolles." (Beckett, Fin de Partie, 1957, p. 45) 
En effet, les indices du temps sont fort remarquables sur les personnages de Beckett à savoir Pozzo qui, à cause de l'abolition de tous les indicateurs du temps de la pièce, ne peut pas se rappeler ce qui s'est passé hier:

\section{"Pozzo : Je ne me rappelle avoir rencontré personne hier.}

Mais demain je ne me rappellerai avoir rencontré personne aujourd'hui" (Beckett, En attendant Godot, 1953, p. 130)

En effet, hier, il y avait tout ce qui ne se trouve pas aujourd'hui, il y avait la mer, le soleil et les sables de la plage. Hier, Nagg et Nell, dans Fin de Partie, avaient des jambes, mais maintenant ils sont culs de jatte. Hamm était en bonne santé, maintenant il est cloué sur un fauteuil à roulettes. Clov ne souffrait ni de hémorroïdes ni de colonne vertébrale, maintenait il est paralytique.

En somme, avec le temps immobile et monotone, les personnages sont condamnés à la même répétition de mouvements, et avec la destruction de toutes les étapes temporelles, ils suivent toujours le même ordre et le les mêmes rites. À titre d'exemple, La vie de Hamm est, dans Fin de Partie rituelle. Il commence la journée avec son réveil et se termine avec son coucher, ce qui amène à une grande monotonie aux actions qu'il fait chaque jour.

Par ailleurs, Beckett, pour augmenter l'embarras chez les personnages et dégrader toute illusion référentielle du temps, détruit les indicateurs temporels. Le temps ravage peu à peu leurs corps, mais sans être perçu comme le cancer qui sévit malignement dans le corps de l'être humain, et c'est en cela que réside la technique dramatique de Beckett : la lumière est devenue grisâtre, le jour est aussi comme la nuit, les deux sont égaux. 
"Quand viendra Godot? Vladimir propose un jour, Estragon en doute, puis l'absence de repère présent dénonce toute possibilité de fixer une date :

Vladimir : il a dit samedi (un temps) il me semble.

Estragon : Apres le turbin

Vladimir : J'ai dû le noter (il fouille dans ses poches archibondées de saletés de toutes sortes)

Estragon: Mais quel samedi ? Et sommes-nous samedi ? Ne serait-on pas plutôt dimanche ? Ou lundi ? Ou vendredi ?' (Beckett, En attendant Godot, 1953, p. 19)

Afin de valoriser cette incertitude, Beckett la met face à face au souci des personnages pour marquer une date ou une heure : comme le demande Hamm sans attendre une réponse: "On est en quel mois ?'.Pozzo regarde sa montre et « s'interroge sur l'heure » (Beckett, En attendant Godot, 1953, p. 19).

Pour ces personnages, le passé n'a produit aucune certitude et le futur n'est plus certain, donc ils s'énervent lorsqu'on leur demande n'importe quel indice temporel. Selon eux, la monotonie du temps va en paire avec l'immobilité de leur corps.

Pozzo s'énerve lorsque on le questionne sur l'avenir et sa réponse s'adresse autant à Vladimir qu'aux spectateurs :

"Vous n'avez pas fini de m'embêter avec vos horaires du temps", (Beckett, En attendant Godot, 1953, p. 127)

Tandis que, dans Fin de Partie, le problème du temps est résolu dès le début de la pièce. "Hamm : Quelle heure est-il ?

Clov : la même que d'habitude 
Hamm : Tu as regardé?

Clov : oui

Hamm : et alors?

Clov : Zéro" (Beckett, En attendant Godot, 1953, p. 130)

4- La chute du corps

Le traitement du corps dans le théâtre beckettien vise à produire une sorte d'anticorps théâtral. (Hubert, Langage et Corps fantasmé dans le théatre de Samuel Beckett, 1970, p. 40) Ce dernier est considéré comme l'une des techniques les plus importants du théâtre de l'absurde. La technique de Beckett réside dans la déformation du corps des personnages en vue de les séparer de toute référence humaine. Chez le dramaturge, plus que les stigmates sont énormes chez les personnages, plus que le message serait visé chez les spectateurs. Ses acteurs sont censés mettre en scène une corporéité plus que l'incarnation même du rôle.

L'analyse d'une mise en scène d'un corps chétif exige que le dramaturge renonce de toute signification de la valorisation de l'homme afin de mieux représenter sa vision désespérée de la condition humaine. Car ses pièces ne représentent pas des corps souffrants ou handicapés par la nature. Il joue sur la dimension corporelle par le biais de la dramaturgie. Selon lui, la chair des acteurs ne s'humanise pas les figures beckettiennes, elle doit être insérée dans un espace d'infirmité et d'amoindrissement en vue de mieux interpréter sa vision absurde du monde.

Cet anticorps théâtral élimine ce qu'importe la vision positive et progressiste du corps. Il est, par exemple dans Oh les beaux jours, humilié, démantelé, ramené vers le sol, et même vers le sous -sol. Le 


\section{Dr. Mohamed Abdel Tawab}

corps s'y oppose à l'image de l'homo erectus (Hubert, Langage et Corps fantasmé dans le théatre de Samuel Beckett, 1970, p. 43) qui désigne le stade de l'hominisation durant lequel l'homme a acquis le statut vertical. $\mathrm{Au}$ lieu d'avoir un corps qui grandit et qui marque ses territoires, il rétrécit et s'abaisse.

Beckett nous met en évidence un corps qui n'évolue pas, il va ainsi en contradiction avec les principes de l'humanité et les récits anthropologiques de l'homme. Sa technique contribue à empêcher ses personnages de tout avancement dans la vie en les dénuant de leurs moindres manifestations et de leurs poids d'expérience. Les indications scéniques suggèrent de rompre avec un corps instrumental, qui concrétise la volonté humaine, nous pouvons l'éprouver dans Fin de Partie où" Vladimir marche à petits pas raides" (Beckett, Fin de Partie, 1957, p. 10),Estragon boite (Beckett, Fin de Partie, 1957, p. 17), Clov a la démarche raide et tremblant (Beckett, Fin de Partie, 1957, p. 15) .Lucky est attaché à une corde, Hamm est condamné à être cloué tout le temps sur son fauteuil ,Nag et Nell sont enterrées dans des poubelles. L'extrême contrainte physique imposée par Beckett oblige à faire le deuil de l'expressivité théâtrale, et mène à transmettre l'intérêt du spectateur vers un corps fragmenté.

De plus, l'infirmité fréquente dans les œuvres dramatiques de Beckett met l'accent sur la déchéance humaine qui joue sur l'immobilité des corps et sa faiblesse progressivement au fil du temps, afin d'en retirer finalement les ultimes potentialités. Cela se voit à travers la fonction des handicaps dont les personnages sont affaiblis. Estragon à la jambe blessée. Luky se croit être enfoncé et embarrassé dans un filet, Hamm est paralysé, Clov ne peut s'asseoir, Nagg et Nell ont leurs jambes dans un accident de tandem. Le processus d'amoindrissement poursuit son 
cours, de sorte que le corps ne cesse pas de s'aggraver : "Hamm : j'ai toujours mal aux jambes" (Beckett, Fin de Partie, 1957, p. 55).

Les facultés des personnages diminuent peu à peu, ce que le décor met en relief dans Fin de Partie par le biais du déséquilibre entre les corps et les fenêtres trop hautes. :"Tu as rapetissé ?" (Beckett, Fin de Partie, 1957, p. 45), demande Hamm à Clov qui va chercher l'escabeau.

La chute est donc le sort prévu des corps sur l'espace scénique comme s'il était naturel d'être né infirme et malade, c'est pour cela que les personnages commentent parfois positivement cet effondrement:"Ce qu'on est bien par terre" (Beckett, En attendant Godot, 1953, p. 118), observe Estragon.

En attendant Godot décline les figures de cet effondrement "titubent la scène" (Beckett, En attendant Godot, 1953, p. 99), Lucky "s'abaisse lentement, jusqu' à ce que la valise touche le sol, se redresse brusquement, recommencer à s'incliner" (Beckett, En attendant Godot, 1953, p. 35).

À la fin de la pièce, Pozzo et lui restent couchés au milieu des bagages"' (Beckett, En attendant Godot, 1953, p. 110).

Selon Beckett, Ce recours à l'affaissement des corps ne suffit pas pour mettre en valeur le côté tragique de la destinée de l'homme, il réalise aussi un processus physique sur les ruines du portrait de l'homme en pied.

De plus, En présentant des personnages déjà tombés, Fin de Partie approuve le mouvement et le seul à se déplacer regrette de n'avoir pas atteint cette position :

"Quand je tomberai je pleurai de bonheur" (Beckett, Fin de Partie, 1957, p. 110), déclare Clov. Jamais, la chute n'aurait pas constituée pour 
Beckett une allégorie tragique ou un rappel biblique. La preuve en est que les personnages peuvent parfois se remettre debout alors que le spectateur les croyait complètement effondrés. Lucky est tout le temps debout, et Vladimir, qui n'y arrive jamais, supplie Estragon de l'aider afin de pouvoir reprendre son équilibre :'"Estragon : si on se levait, pour commencer?

Vladimir : Essayons toujours.

Ils se lèvent

Estragon : pas plus difficile que ça.

Vladimir : Vouloir, tout est là' (Beckett, En attendant Godot, 1953, p. 109)

En gros, tomber ou se lever de nouveau sont des figures qui appartiennent aux possibilités scéniques. Pour cette raison, les personnages les accomplissent de manière incertaine.

La décision ne dépend pas de la volonté individuelle de chaque personnage. Elle présente l'essai d'une posture. Cette alternance entre le lever et le fait de tomber représente l'aspect régulier de la chute : elle laisse apparaitre un engagement dramaturgique établi par Beckett, une ligne principale qui impose un mouvement descendant. Elle s'insère dans un processus d'immobilisation qui accable corps. Fin de Partie présente cette technique dramatique comme une nécessite quand Hamm prend le ton de prophète et prédit :

"Un jour, tu te diras, je suis fatigué, je vais m'asseoir, et tu iras t'asseoir. Puis tu te diras, j'ai faim, je vais me lever et me faire à manger. Mais tu ne te lèveras pas. Tu te diras, j'ai eu tort de m'asseoir, mais puisque je me suis assis je vais rester assis encore un peu, puis je me lèverai et je me ferai à manger. Mais tu ne te lèveras pas (.....) (Beckett, 
Fin de Partie, 1957, p. 110).Et lorsque Clov lui rappelle l'accord de départ qui l'empêche de s'asseoir, Hamm monte en lui disant qu'il se couchera, «Qu'importe la posture ! (Beckett, Fin de Partie, 1957, p. 58), et que de toute façon qu'il faut chuter. La formule résume le principe beckettien

Pour conclure, chaque pièce met en scène différentes positions du corps dont aucune n'a de signification privilégiée : elles se répètent, s'opposent, se déroutent et se croisent .C'est de cette manière qu'elles amoindrissent les poses théâtrales du corps et les dénoncent comme des impostures.

\section{5-Le corps chétif est une arme à double}

\section{tranchante :}

Dans l'univers de Beckett, nous voyons que le corps infirme prend deux aspects : une fois, il est ressenti comme tragique et d'autre fois comme comique et c'est là qui réside son esthétique dramatique pour attirer finalement l'attention des lecteurs, d'une part et les spectateurs d' autre part.

5-Le corps chétif est une arme à double tranchant :

Dans l'univers de Beckett, nous voyons que le corps infirme prend deux aspects : une fois, il est ressenti comme tragique et d'autre fois comme comique et c'est là que réside son esthétique dramatique pour mieux agir sur l'attention des lecteurs et les spectateurs

5-1Le corps chétif est un ressort tragique

D'abord, nous allons voir comment le corps est représenté comme tragique chez Beckett : le handicap est considéré comme un moyen tragique du théâtre beckettien. La défaillance mentale et physique 


\section{Dr. Mohamed Abdel Tawab}

apparait comme un repère temporel dans le sens où il rappelle le passage du temps et annonce par ses déformations incessantes la mort à venir. Nous pouvons l'éprouver à travers Les gestes quotidiennes difficiles des personnages comme la déambulation difficile ou disharmonieuse, Vladimir dans En attendant Godot marche «à petits pas raides» (Beckett, En attendant Godot, 1953, p. 10), Estragon se déplace « en boitillant » (Beckett, En attendant Godot, 1953, p. 19). Clov de Fin de partie est « raide et vacillante » (Beckett, Fin de Partie, 1957, p. 13)

Chaque tentative d'agir se conclut par un échec, en imputant cet échec à l'impuissance du personnage. «Je ne pouvais littéralement» (Beckett, La Comedie, 1972, p. 21) dit "Estragon, assis sur une pierre, essaie d'enlever sa chaussure. Il s'y acharne des deux mains, en ahanant. Il s'arrête à bout de forces » (Beckett, En attendant Godot, 1953, p. 10). De plus Pozzo n'arrive pas à se relever alors qu'il est à terre. Le corps devient alors un miroir qui concrétise la fin du monde. Nous ne faisons rien qu'attendre la fin, comme le dit Godot«Rien à faire » (Beckett, En attendant Godot, 1953, p. 10).

Donc, nous pouvons saisir que la dimension tragique du corps contribue à faire passer le temps et finalement à l'annonce de la mort.

D'autre part, puisqu'ils sont handicapés qu'ils ne peuvent plus atteindre le statut de l'équilibre. La vieillesse constitue donc ici une sousestimation essentielle du corps. Ce sont aussi des personnages qui souffrent tout le temps d'une fatigue générale et faiblesse totale : «Estragon s'endort. (...) se réveille en sursaut» (Beckett, En attendant Godot, 1953, p. 24)

Beckett présente donc des êtres en déclin physique, suffoqués et qui sont en train de s'agoniser. Par leur aspect chétif, ces personnages portent tous en eux des maux qui les rongent. Le dramaturge dramatise 
avec minutie les traits remarquables de leur infirmité afin d'en accentuer l'aspect tragique .De surcroît, il met au public cette différence avec la norme qui agit comme exigence d'adaptation au monde. Toutefois, Les personnages se montrent souffrants de leur rapport au monde et c'est en cela qu'ils appartiennent au registre tragique.

Dans le même ordre d'idées, Beckett avait recours à ce corps fragmenté pour révéler une nouvelle esthétique propre à lui, il s'agit du « marionnettisme» (Hubert, le theatre, 2008, p. 189). Les personnages supportent aussi d'autres formes d'handicap, c'est l'imputation des parties du corps, ce qui en résulte un arrêt de ses fonctions.

C'est le cas de l'imputation des parties de Nagg à qui Clov demande : «Comment vont tes moignons?» (Beckett, En attendant Godot, 1953, p. 23) L'immobilité physique qui aurait censé être un moteur efficace est sur le point d'arrêter de fonctionner. Les rouages semblent bloqués. Cette paralysie partielle est à la fois un mode d'être et d'agir dans la mesure où le handicap est aussi ce qui permet aux personnages d'entrer en contact les uns avec les autres et de déterminer les finalités d'une action : essayer de se redresser, d'attraper un objet, de partir. Par les dysfonctionnements des parties du corps, cette « mécanique déréglée » (Brenner, 2007, p. 952) ; comme l'écrit Jacques Brenner, Beckett vise à rendre ses personnages comme des pantins misérables soumis aux cordes du dramaturge qui les garde en déséquilibre. Cette désorganisation et ce démembrement exigent la gravitation des corps en faillite puisque le corps semble ne plus appartenir au personnage lui-même.

L'esprit a subi aussi ce balbutiement. Les paroles des personnages ne sont plus cohérentes, reliées entre elles par un fil logique qui les 


\section{Dr. Mohamed Abdel Tawab}

organise. La déchéance mentale est mise en scène comme véritable minimisation de l'esprit.

Par ailleurs, Beckett, pour mettre l'emphase sur l'aspect tragique chez ses personnages, est allé plus loin jusqu'à les incarcérer afin de les déshumaniser et accentuer ce drame devant le public. Donc, nous pouvons dire que c'est une autre forme d'handicap: C'est le cas de certains personnages totalement privés de leur mobilité. Beckett a recours de l'incarcération, et de l'impossible mobilité du corps. Nagg et Nell sont enfermés dans des poubelles. . Cette privation de mobilité est évidemment très marquée dans Fin de partie où Hamm est en fauteuil roulant : «Je ne peux pas m'asseoir » dit Clov, ce à quoi Hamm répond «Et moi je ne peux pas me tenir debout » (Beckett, Fin de Partie, 1957, p. 28). De surcroit, les personnages de Comédie sont enfermés dans des jarres et Winnie est quant à elle enterrée dans le sable jusqu'à la taille. La diminution du corps beckettien est d'autre part toujours associée à une minimisation de l'esprit, comme en témoigne la remarque de Clov: "J'ai mal aux jambes, ce n'est pas croyable. Je ne pourrai bientôt plus penser » (Beckett, Fin de Partie, 1957, p. 68)

On retrouve d'autres handicaps comme l'aveuglement. En effet, aux questions «comment vont tes yeux? », Clov répondra «mal» (Beckett, Fin de Partie, 1957, p. 25), échange quasiment similaire entre Nagg et Nell«tu me vois? Mal.», "Et toi tu me vois ? Mal Hamm rappelle à Clov «Un jour tu seras aveugle. Comme moi » (Beckett, Fin de Partie, 1957, p. 33). De plus, d'un point de vue symbolique, les problèmes de vue, quels que soient leur degré, attestent de l'aveuglement progressif ou total des personnages, voire soulignent une volonté de s'aveugler pour échapper de la condition humaine dans laquelle ils 
vivent. Enfin, nous ne pouvons dire la figure de l'aveugle est prioritaire, ce qui motive le tragique chez Beckett.

D'autre part, le handicap mental apparaît chez Beckett comme un facteur d'emprisonnement. Les personnages qui présentent des troubles obsessionnels compulsifs sont nombreux et s'enferment dans la répétition de gestes dérisoires, témoignant de l'impossible développement d'un corps.

Nombreux sont donc les éléments qui emprisonnent les personnages sur la scène en raison de leur impotence, ce que souligne James Knowlson au sujet de Godot dont les personnages sont des «être enfermés dans des cages »(Knowlson, 1990, p. 521). Cette prison s'érige en dramaturgie de la boucle, en métaphysique du recommencement. L'incarcération semble être le thème dominant.

Il y a enfin, chez certains personnages Beckettiens, une sorte de déshumanisation qui les transforme en des êtres serviles : Pozzo finit par s'éloigner, au deuxième acte, «en rampant» (Beckett, En attendant Godot, 1953, p. 118). De plus, Winnie dit à Willie «Oh je sais bien, mon chéri, ramper à reculons, ce n'est pas de tout repos » (Beckett, Oh!les beaux jours, 1963, p. 32). Cette dépravation d'humanité n'est qu'une autre forme de la condition tragique des personnages beckettiens.

Pour autant, malgré la représentation des scènes tragiques des personnages beckettiens, cela n'empêche pas de dire qu'il les a créées pour en engendrer l'aspect comique qui permet de revaloriser véritablement la sous-estimation du corps pour en faire un élément de gaieté et de compassion comme signe de solidarité avec les personnages.

- Le corps chétif et mental estun ressort comique 
Si nous portons un regard sur le côté comique dans l'univers théâtral de Beckett, nous constatons que les handicaps physiques sur l'espace scénique interprètent une puissante dimension comique.

Le discours même des personnages devient le lieu d'échanges burlesques que l'on peut intégrer au ressort comique de mots, comme le témoigne les répliques entre Nagg et Nell : « Notre ouïe n'a pas baissé » dit Nagg. « Notre quoi ? » (Beckett, Fin de Partie, 1957, p. 24) lui répond Nell.

De plus, le vieillissement est également une source vitale chez le dramaturge, il lui permet également de libérer le comique, comme en témoigne ce dialogue entre Nagg et Hamm :

Nagg : Ma bouillie!

Hamm : Maudit progéniture!

Nagg : Ma bouillie!

Hamm : Ah il n'y a plus de vieux! Bouffer, bouffer, ils ne pensent qu'à ça' ' (Beckett, Fin de Partie, 1957, p. 44)

Les difficultés mentales liées au vieillissement témoignent également de cet ancrage dans le registre comique.

Enfin, les pathologies mentales font allusion aussi à ce registre comique. La tirade de Lucky en est l'exemple le plus marquant. Sa jargonaphasie est très drôle et incite le rire de tout le monde.

La représentation des empêchements physiques peut aussi être évoquée par bien des aspects et des scènes clownesques qui contribuent finalement au ressort comique à savoir dans leur gestualité, les personnages apparaissent non seulement comme des clowns mais aussi comme des gens qui souffrent des retardements mentaux, cela constitue 
pour Tristan Rémy, historien du cirque, un élément essentiel dans la figure du clown. La réplique de Vladimir, "C'est peut-être un idiot» (Rémy, 1962) qui précède celle d'Estragon, «Un crétin » (Beckett, En attendant Godot, 1953, p. 35) met en relief cette expression de figure clownesque

Le corps défavorisé, impotent et maladroit, est un des aspects du comique de cirque. Ces difficultés de la marche sont à l'origine de jeux scéniques inspirés des clowns. D'ailleurs, nombreuses sont les pièces de Beckett qui sont mises au public sur ce mode. Premièrement, nous pensons à la mise en scène de Roger Blin en 1953, qui écrit :

Ma première idée quand j'ai lu le texte a été de représenter les personnages dans un cirque. Parce que les rapports entre Vladimir et Estragon, la rapidité de leurs répliques m'ont tout de suite fait penser à des dialogues de clowns [...] (Chabret(dir), 1990, p. 167)

Mais cette représentation ne passe pas sous silence sans être mise en critique, Alain Satgé critique ce type du clown :

"Beckett a choisi le style du cirque. Ses deux héros sont pareils, dans leur redingote réparée et sous leur vieux melon cabossé, à deux augustes qui évoluent sur la piste de sciure" (Satgé, 1999, p. 77)

D'autres metteurs en scène ont pris comme source d'inspiration la dimension clownesque de Samuel Beckett pour sa mise en scène; Évoquons par exemple, Marcel Maréchal, quand il décide de représenter Fin de partie en 1964 à Lyon, s'inspire de la dimension clownesque de Samuel Beckett pour sa mise en scène. Lorsqu'il évoque ses choix, il rappelle la nécessité du «maquillage superbe à la Fratellini », et le jeu de scène des acteurs : 


\section{Dr. Mohamed Abdel Tawab}

«Cela a été joué avec énormément d'humour, comme il fallait que ce soit joué, comme Grock ou Laurel et Hardy l'auraient joué » (Maréchal, 19990, p. 207)

Les personnages de Beckett ont donc l'allure du clown-robot, réalisant ainsi des mouvements répétitifs comme les marionnettes. Cet acte de la répétition est la première source du comique, comme le souligne Alain Satgé :

"La séquence commence comme un numéro de cirque: les visiteurs exécutent une entrée de clowns. Pozzo avec son fouet évoque aussitôt l'image du dompteur, tandis que Lucky fait penser tantôt à un cheval, tantôt à un chien ou à un ours savant'. (Satgé, 1999, p. 206)

Chaque nouvelle répétition est alors source de comique, car elle ne fait que souligner le décalage entre l'action et la catastrophe, la succession d'échecs qui rendent son accomplissement impossible. La ritualisation des catastrophes est un élément essentiel dans le théâtre beckettien. Prenons par exemple; Clov déplace le fauteuil roulant de Hamm « un peu plus à gauche », « un peu plus à droite » et cela plusieurs fois d'affilé dans la pièce, tout comme la chaussure qu'Estragon essaie d'enlever «il recommence », « renonçant à nouveau », « remet », « passe sa main à nouveau » (Satgé, 1999, p. 211)

Puisque le déboitement peut être vu comme un facteur de tragique, cela n'empêche pas de dire qu'il est associé au comique. Le clown qui se glisse dans le personnage d'un pantin boiteux est un motif pour le rire et l'amusement.

Pour conclure nous pouvons dire que le corps impotent voir tragique a deux faces : Il a recours au ressort tragique pour susciter la pitié et la compassion chez les spectateurs, d'une part, et le côté comique pour atténuer la pesanteur tragique. Donc, les personnages vivent dans 
une véritable ambivalence entre le comique et le tragique. Cette technique leur permet de dépasser cette impossible solitude totale.

\section{Conclusion}

$\mathrm{Au}$ terme de notre recherche, nous constatons que la place que donne Beckett au corps handicap, chétif ou impotent est prioritaire dans le théâtre de Beckett.

La nouveauté chez Beckett, c'est qu'il met le corps de l'être humain en question, dans une brutale interrogation très angoissante. Tout au long de l'œuvre dramatique de Beckett, nous voyons que le corps chétif est représenté attaché dans une machine qui tourne à vide, point de départ de toute métaphysique. Selon ce dramaturge, la mise en scène d'un corps souffrant des troubles physiques et mentales révèle d'une humanité, quoiqu'agonisante, résiste encore et attache à la vie comme s'il était normal d'être né handicap ou fou dans la vie.

L'importance dramatique et philosophique de Beckett réside dans sa technique à mettre en scène l'image du corps d'une manière primitive afin de donner l'occasion aux spectateurs de comprendre le rapport que le personnage entretient entre son âme et son corps. Beckett voulait présenter au public un personnage qui peut contrôler son corps chétif et handicap, même s'il était vide de l'intérieur. De plus, Beckett a adopté le thème du corps dans son art théâtral, car il a un une valeur mythique, c'est-à-dire qu'il est censé être un instrument qui tente de mettre en scène un corps imaginaire dans la peau d'un personnage qu'est le comédien, homme en chair et en os. Selon le dramaturge, nous ne pouvons apercevoir les choses qu'à travers l'imagination. Donc, nous pouvons dégager que la représentation d'un corps malade et son rapport avec le personnage demeure toujours un domaine de l'imaginaire dans le théâtre de Beckett. D'autre part, nous avons vu que Beckett pratique l'inversion 
des données. L'anormalité devient la normalité, l'illogique devient logique, l'insignifiance devient signifiance, la bizarrerie devient alors la norme dans le théâtre de Beckett. Tout cela n'a qu'un seul objectif : interpréter l'absurdité de la condition humaine dont le pessimisme, le handicap et l'immobilité des personnages en sont un exemple.

Même l'échec devient quelque chose de normal, toute tentative d'échec se voue à l'échec comme le montre cette citation : «Échoue encore. Échoue mieux » (Martin, 1971, p. 55). Cette poétique de l'échec est omniprésente dans son théâtre. Mais c'est un échec qui se veut en victoire puisqu'il devient l'expression de la persévérance.

D'ailleurs, nous avons éprouvé que le duo est une partie intégrante dans l'univers beckettien, chacun ne peut pas se passer de l'autre. Il est le moyen de l'amusement chez le handicap et la condition de la survie des couples. Les corps sous-estimés deviennent outils de lutte solidaire contre l'adversaire : le temps, et l'attente de la mort. Que ferait Winnie sans Willie? Que deviendrait Hamm sans Clov?

Beckett a recours aussi au ressort comique pour atténuer l'effet du côté tragique chez ses personnages.

Pour conclure, nous pouvons dire que la déficience physique et mentale est un bienfait pour les personnages beckettiens et que toute envie de mobilité et de guérison serait une malédiction:"quelle malédiction la mobilité " (Beckett, En attendant Godot, 1953, p. 33) 
La représentation dramatique du corps chétif dans ... 


\section{Dr. Mohamed Abdel Tawab}

\section{Bibliographie}

Artaud, A. (1966). le theatre et son double. Paris: Gaiimard.

Beckett, S. (1953). En attendant Godot. Paris: Editions de Minuit.

Beckett, S. (1956). le Calmant. Paris: Edition de Minuit.

Beckett, S. (1957). Fin de Partie. Paris: Editions de Minuit.

Beckett, S. (1963). Oh!les beaux jours. Paris: Editions de Minuit.

Beckett, S. (1972). La Comedie. Paris: Editions de Minuit.

Beckett, S. (1972). Pas moi. Paris: Editions de Minuit.

Beckett, S. (1978). fragment du theatre I et II. Paris: Esquisse Radiphonique.

Brenner, J. (2007). Samuel Beckett,En attendant Godot. Dossier de presse, 952.

Chabret(dir), P. (1990). Samuel Beckett. Revue d'esthétique, 167.

Chabret, P. (1986). Samuel Beckett. Revue d'esthetique, N0 521.

Dodge, D. (1968). le couple Dodge. Paris: Hachette.

Foucré, M. (1970). Le Geste et lq parole dans le théatre de Beckett. Paris: Nizet.

Freud, S. (1983). Métapsyhchologie. idées Gallimard.

Fuentès, C. (1972). le borgne est roi. le theatre 140, 3.

Grossman, E. (Novembre 1998). l'esthétiaque de Beckett. Paris: Editions de Sedes.

Hubert, M.-C. (1970). Langage et Corps fantasmé dans le théatre de Samuel Beckett. Paris: Nizet.

Hubert, M.-C. (2008). le theatre. Paris: Armand Colin.

Knowlson, J. (1990). Samuel Beckett. Revue d'esthetique, 521.

Léautier, G. (1978). la Matriarche. Paris: Compagne première.

Liègme, B. (1976). Tandem. Lausanne: Hauterive.

Maréchal, M. (19990). Samuel Beckett. revue d'esthétitique, 207.

Martin, E. (1971). le theatre de l'Absurde. Paris: Buchet-Chastel.

Mrozek, S. (1961). Striptease. Dialogue, N0 6.

Picasso, P. (1964). Le desir attrapé par la queue. Paris: Blanche. 
Rémy, T. (1962). Entrées clownesques. Paris: l'Arche.

Ryngaert, J. P. (1993). Lire En attendant Godot. Paris: Dunod.

Satgé, A. (1999). Samuel Beckett,En attendant Godot. Paris: Presse universitaire de France.

\section{Bibliographie}

Le corpus

1-Samuel Beckett, En attendant Godot, Editions de Minuit, 1953

2-Samuel Beckett,Fin de Partie,Editions de Minuit, 1957

3-Samuel Beckett, Oh ! Les beaux jours, Editions de Minuit, 1963 


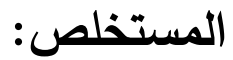

تقوم الدراسـة في إطار مسرحي حول رؤية الكاتب المسرحي سماويل بيكيت لهذها الويلات التي نجمت عن هذالحروب من خلال تمثيل الكاتب للجســ الواهن في بعض مسـرحياته ,نذكر علي سـبيل المثال:في انتظار جودوو, الايام الجميلة ونهاية اللعبة نتبع في هذا البحث الطريق التحليلية من اجل كثـف الجانب المخفي من الثــصـية علي خثـبة المسـرح للجمهور .يقوم بيكيت من خلال روؤيتة المسرحية في عرض الجسد الواهن علي أشكالi المختلفة: الجسد المدمر بالوقت, الجسد الواهن وايثرة الكوميدي علي الجمهور , الجســــــ الواهن واثره التراجيدي علي الجمهور ـ وفي ختام البحث توصـــلت الاراسة الي ان تمثيل الجسد الواهن ماهو الي تعبير بعد الحرب العالمية الاولي والثانية وإلقاء الولايات المتحدة قنبلتيها النووية علي جزيرتي هيروشـيما ونجازكي, ظهر تيارأ يســي التيار" العبثي". من اهم رواد هذا التيار نذكر: جون اينسـكووالبير كامي وكاتبنا المسـرحي سـامويل بيكيت وهذا الاخير يمثل محور دراسـتنا.عن الحالة العبثية وحالة اللا مبالاة التي يتصـف بها الكاتب خاصــة بعد هذه الويلات

$$
\text { التي نجمت عن الحروب. }
$$

الكلمات الدالة: الجسد الواهن - العبثية- سامويل بيكيت- المأساة. 